\title{
Enhancing planned and associated biodiversity in French farming systems
}

\author{
Marion Casagrande ${ }^{1,2} \cdot$ Lionel Alletto $^{3,4} \cdot$ Christophe Naudin $^{5} \cdot$ Arthur Lenoir $^{1}$. \\ Ali Siah ${ }^{6}$ - Florian Celette ${ }^{1}$
}

Accepted: 27 September 2017 /Published online: 30 October 2017

(C) INRA and Springer-Verlag France SAS 2017

\begin{abstract}
In a context of global change involving uncertainty in agricultural production, agroecological systems need to reduce their dependency on inputs and increase their resilience. Biodiversity-based techniques are promising, as they provide production services based on biological processes. Tracking farmer practices is an original approach aiming at identifying and analysing alternative systems and supporting the development of these techniques. We studied, for the first time, the onfarm implementation of six biodiversity-based techniques: (i) agroecological infrastructures, (ii) cropped varietal mixtures, (iii) agroforestry, (iv) intercropping, (v) cover cropping and (vi) crop rotation diversification. We first analysed the combinations of these techniques in a large sample of 194 French farmers. A multiple correspondence analysis followed by a hierarchical cluster analysis on principal components resulted in groups of farms with different combinations of these techniques. Then, deeper interviews were conducted with 29 farmers across three regions to analyse the various methods
\end{abstract}

Florian Celette

fcelette@isara.fr

1 ISARA-Lyon (member of the University of Lyon), Department of Agroecology and Environment, 23 rue Jean Baldassini, 69364 Lyon cedex 07, France

2 ITAB, Quartier Marcellas, 26800 Etoile sur Rhône, France

3 Université de Toulouse, INP Purpan, UMR AGIR, 75 voie du TOEC, 31076 Toulouse, France

4 CRA Occitanie, BP 22107, 31321 Castanet-Tolosan, France

5 USC LEVA, ESA (Ecole Supérieure d'Agricultures-Angers Loire)-INRA-SFR 4207 QUASAV, Univ. Bretagne Loire, 55 rue Rabelais, 49007 Angers, France

6 Institut de Recherche Charles Violette (EA 7394)-Equipe Adaptation aux Stress et Qualité des Végétaux, ISA Lille, 48 bd Vauban, 59046 Lille cedex, France of applying the techniques in the context of their farm and to identify the conditions for their successful implementation. Taking advantage of this large and rare sample of almost 200 interviewed farmers, we identified six different groups of farms. From farms applying mainly cover cropping to comply with European regulations to much diversified farms implying the redesign of the farming system, we support the idea that different strategies of implementation of such biodiversitybased techniques co-exist. The in-depth interviews demonstrated that the level of diversification is related to farm characteristics and four factors mainly favoured the development of such techniques on farms: (1) available labour force and (2) specialised machinery (internal factors) as well as (3) access to market opportunities and (4) the exchange of knowledge through networking (external factors). Surprisingly, the conservation agriculture farmers of our sample did not apply significantly more biodiversity-based techniques. However, our results indicated that organic farmers applied significantly more of these techniques. Our results suggest that enhancing knowledge exchange through networks would favour the broader application of such techniques. It could also be relevant to gather farmers, industries and public authorities to favour the emergence of market opportunities.

Keywords Agroecology · Crop diversification · Farmer practices $\cdot$ Survey $\cdot$ Cover cropping $\cdot$ Intercropping

\section{Introduction}

In a global change context, agricultural production has to face an increasing uncertainty concerning quantity and quality of production. At the same time, agricultural production is largely subjected to climate change and price variation (Tomich et al. 2010). Increasing the resilience of agroecosystems, i.e. 
the capacity of a system to recover its original structure and function after a disturbance (Tomich et al. 2010), could therefore maintain the provision of vital services such as food production even in the case of unpredictable climatic or economic hazards (Lin 2011). Moreover, reducing the dependency of the cropping systems on exogenous inputs may help the stabilisation of the economic performance of the copping systems in the context of price variation. Reduced dependency is also one of the keys to achieve system resiliency, permitting to maintain or even increase food production while limiting environmental impacts (Tilman et al. 2002).

Agroecological systems can rely on biological and ecological processes and traditional knowledge rather than on inputs (Altieri et al. 2011; Wezel et al. 2014). There is a need and demand for the development of agroecological farming systems worldwide. In France, the Ministry of Agriculture initiated an "Agroecological project for France" in 2012 and implemented public policies to favour agroecological systems. This project is largely based on improved knowledge of what some innovative farmers already do and why they do it. Farmers indeed provide innovations combining empirical and scientific knowledge in the design of their systems (Altieri et al. 2011). Tracking on-farm innovations is a concrete approach for identifying and analysing alternative systems (Salembier et al. 2016). Gaining insights into the factors for applying agroecological techniques will support the spreading of agroecological systems. When a technique is applied to a farm, the characteristics of the farm (e.g. soil and climate conditions, available resources, knowledge and objectives of the farmer) shape how the farmer applies the technique, transforming it into a "practice". In other words, a practice is the application by a farmer of a technique adapted to the context of his/her farm.

Agroecological practices are defined as agricultural practices aimed at producing sufficient amounts of food while valorising ecological processes and ecosystem services in the best way instead of using external inputs (Wezel et al. 2014). Such agroecological practices can be classified according to the efficiency substitution redesign (ESR) framework reported by Hill and MacRae (1995). Efficiency refers to improved input use. Substitution practices include substituting chemical inputs with organic inputs or with alternative practices. Finally, redesign refers to a change in the whole cropping system or even the whole farming system.

The improvement of natural and cropped plant diversity at the farm scale is expected to improve ecosystem services (including yield) and, in turn, reduce input use (Finckh and Wolfe 2006; Tomich et al. 2010; Kremen and Miles 2012; Duru et al. 2015; Ponisio et al. 2015) and better cope with climate change (Lin 2011; Naudin et al. 2014). Nevertheless, the development of biodiversity-based agriculture most often requires redesigning farming systems (Duru et al. 2015). Thus, identifying innovative farming practices and the conditions of their successful implementation could help practitioners to further develop such agroecological techniques.

In the present study, we focused on agroecological techniques relying on the services of biodiversity, and we further refer to them as "biodiversity-based techniques". These techniques included those improving both (i) natural biodiversity due to agroecological infrastructures such as hedgerows, fallows and grass strips and (ii) crop biodiversity in time and/or space due to varietal mixtures, agroforestry, intercropping (Fig. 1) cover cropping and diversified crop rotation.

The adoption of biodiversity-based techniques has been limited thus far because (i) input use is not restricted for most farming systems, (ii) policy incentives are oriented towards mono-cropping systems under intensive management, (iii) biotech solutions such as drought-resistant crops have been developed and (iv) sole crop systems are believed to produce more biomass compared with multispecies systems (Lin 2011). Moreover, applying agroecological practices does not only require technical knowledge; how farmers include such innovative techniques in their system highly depends on internal (pedoclimatic conditions, labour force, machinery and capital) and external (availability of inputs, selling products, networks etc.) factors. The challenge is therefore to find the appropriate balance of diversification within the farm system to satisfy both production and protection values (Lin 2011).

Three decision levels at the farm could be considered: (i) at the field scale, the farmer makes tactical decisions regarding annual crop management; (ii) at the cropping system scale (rotation), the farmer makes strategical decisions regarding crop allocation; and (iii) at the farm scale, the farmer allocates strategic production resources. When studying the implementation of new techniques to the farm, it is thus relevant to assess the farmers' room for manoeuvre, i.e. the willingness to adopt technical changes at a particular level without endangering farm system functioning at another level (Navarrete et al. 2006). Depending on the characteristics of the farms

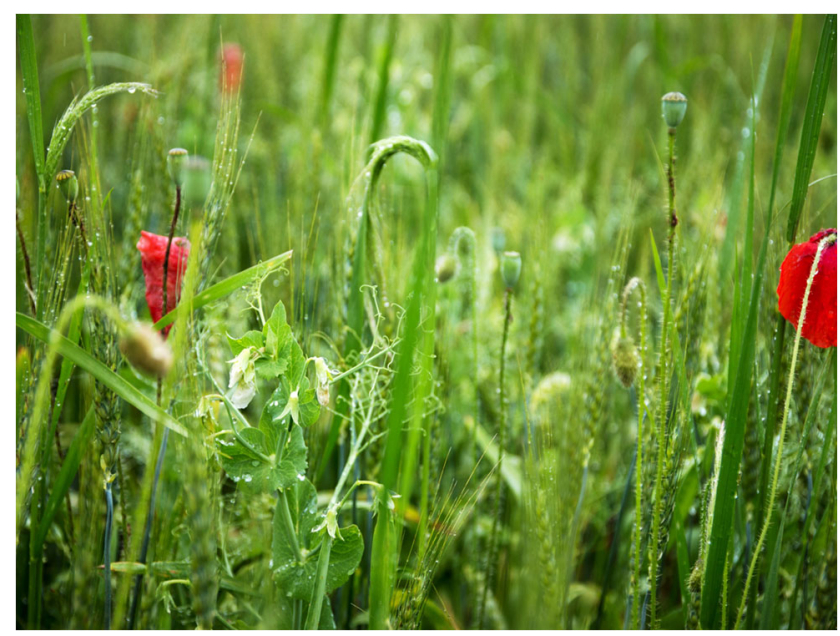

Fig. 1 Intercropping of winter wheat and pea @ Olivier Duchêne 
and on the considered biodiversity-based techniques, the constraints and factors that farmers face might differ. The adaptation and combination of biodiversity-based techniques, i.e. the diversification strategy of the farms, and their scale of application depend on farm characteristics. Indeed, the decision of farmers to implement biodiversity-based techniques is influenced by the ability of the diversification strategy to support the economic resilience of farms (Lin 2011). To fill the gap of knowledge regarding the management of biodiversity-based techniques of farmers and to improve the implementation of such techniques, there is a need for identifying the factors of the application of these techniques on farms in France.

In this study, we tracked on-farm innovations through surveys of 194 French farmers who applied one to six of the abovementioned biodiversity-based techniques in order to identify the conditions for their successful implementation for further development of sustainable farming systems. The objectives were to describe and analyse the diversity of the combinations of techniques among the farm panel and understand the main motivations and goals of the farmers for implementing such techniques. In a second phase, we conducted deeper interviews with 29 of the farmers in order to identify the various practices when farmers adapt the techniques to their objectives and farm organisations. Finally, we identified constraints and factors farmers face when implementing such techniques and discussed the perspectives of larger applications of biodiversitybased techniques.

\section{Materials and methods}

In this study, we focused on six biodiversity-based techniques: (i) varietal mixtures, (ii) agroforestry, (iii) cover crops (including green manure), (iv) intercropping, (v) agroecological infrastructures and (vi) diversified rotations. Varietal diversity relies on intraspecific genetic diversity at the field scale (de Vallavieille-Pope 2004). Agroforestry involves alley intercropping with crops and rows of woody vegetation or scattered fruit trees in meadows (Malézieux et al. 2009; Wezel et al. 2014). Cover crops are sown during the period between two major crops in order to limit environmental impacts (e.g. soil erosion, nitrate leaching) and/or improve soil fertility and/or pest and disease management (Finckh and Wolfe 2006; Wezel et al. 2014). Cover crops are usually not harvested. Intercropping is the growth of two or more species in the same field for a significant period and involves variation in species, respective densities and spatial arrangements (Willey 1979). Agroecological infrastructures encompass the planting and management of vegetation strips and hedges in fields and at field borders (Wezel et al. 2014; Liebman and Schulte 2015). Diversified rotations encompass the production of at least three different crops among fields and across years (Finckh and Wolfe 2006).

\subsection{Tracking on-farm innovations and the first survey of farmers}

This study is based on a sample of 194 French farmers who applied one to six of the previously defined biodiversity-based techniques and who are located within 26 districts in five regions in France. Thirty-seven of these farmers were located in northern France (Nord-Pas-de-Calais region), 76 were in southwestern France (Aquitaine and Midi-Pyrénées regions), 42 were in northwestern France (Pays de Loire region) and 41 were in southeastern France (Rhône-Alpes region). To identify farmers who applied biodiversity-based techniques in each region, we contacted farmer advising services such as agricultural chambers, the "Biodiversity Agriculture Soil and Environment" (BASE) network (i.e. the conservation agriculture network) and groups of organic farmers (GAB). We then carried out a snowball approach to identify a large number of interesting farmers to interview. By tracking innovations, we did not seek to be representative of the French farmer population but rather aimed at identifying on-farm innovations. Consequently, the farm sample of the study maximised biodiversity-based situations and highlighted the diversity and combinations of biodiversity-based techniques, depending on the surveyed farms.

Thus, in the studied farms, the principal type of farming system consisted of arable crops or mixed arable-livestock systems. In the Midi-Pyrénées, Aquitaine, and Nord-Pas-Calais regions, 80,88 , and $97 \%$ of farms were cultivating arable crops as the main production, respectively. In the Rhône-Alpes region, $54 \%$ of the farms had mixed arable-livestock systems and $29 \%$ had arable crops as the main production. In the Pays de Loire region, $88 \%$ were cultivating arable crops as the main production and $50 \%$ of them raised livestock as secondary production. The characteristics of the overall sample and of each regional sample can be found in Table 1 .

We decided to overrepresent the number of organic farmers in the sample compared with national and regional data (Table 1). Indeed, we assumed that organic agriculture may spur farmers to diversify their systems compared with conventional farmers (Navarrete et al. 2014) and to combine biodiversity-based techniques to cope with the absence of chemical external inputs. Nevertheless, organic farmers might be specific in the way they apply such techniques and the constraints they face. We also wanted to gain more information on how organic farmers practise such techniques and identify constraints and factors that could also be relevant for conventional farmers. As we contacted farmers identified using the conservation agriculture network (BASE network), we also overrepresented the number of farmers applying conservation agricultural techniques compared with national and regional data (Table 1). Indeed, as conservation agriculture relies on minimum soil disturbance, maximum soil cover and diversified crop rotations, conservation farmers are likely to apply biodiversity-based techniques. This is an exploratory 


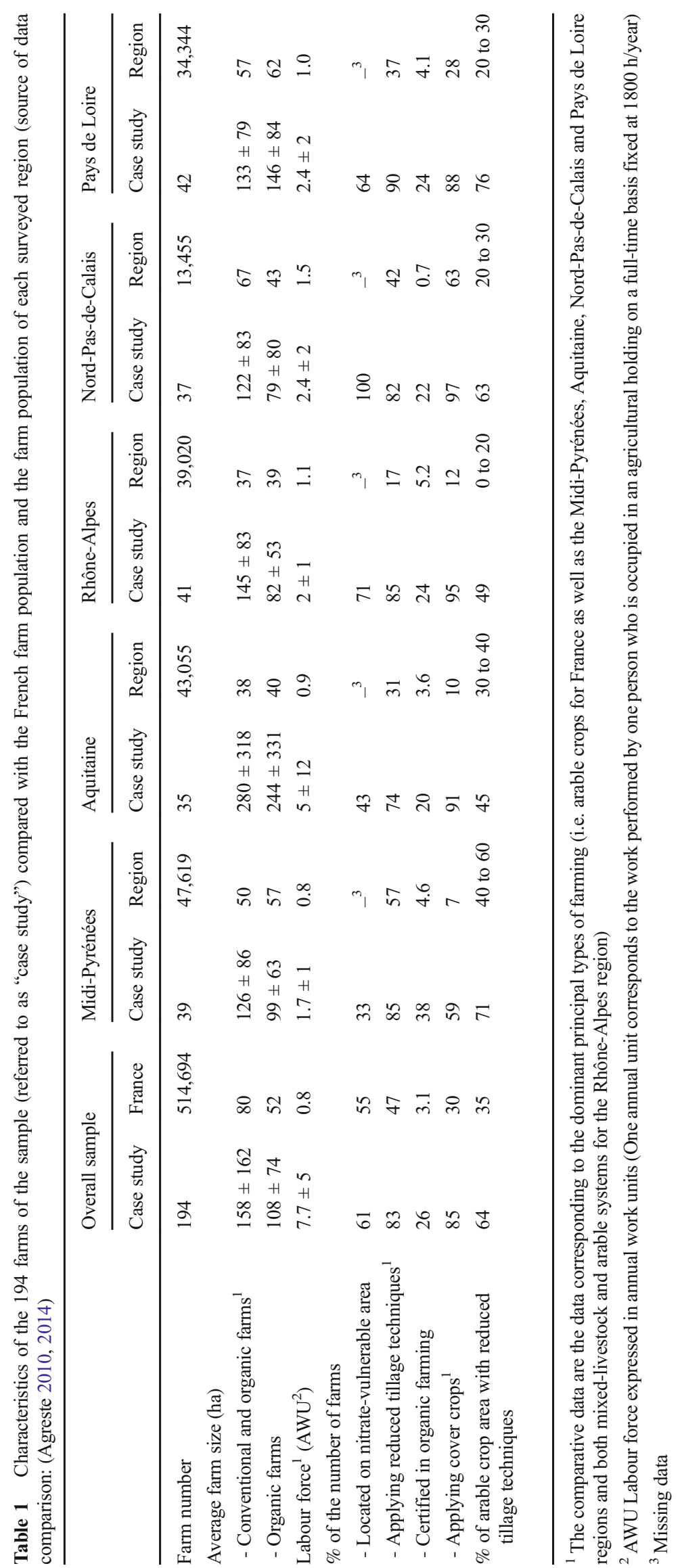


survey that aims to better understand the range of diversity of farmer practices as well as the motivations, constraints and factors for integrating biodiversity-based techniques on their farms. We chose to interview farmers who are rather innovative with respect to the techniques and the way these techniques are applied (Salembier et al. 2016) compared with dominant French arable farming systems.

We conducted phone interviews with questionnaires made of closed-ended questions during winter 2012. The questionnaire was divided into two main sections: (1) general farm description and (2) the application of biodiversity-based techniques. The first part was dedicated to the farm, its environmental description (e.g. farm size, geographical coordinates, soil types and climatic conditions) and its socio-economic characteristics (e.g. principal type of farming, age of the farmer). Moreover, farmers described their cropping systems (e.g. list of crops, crop areas dedicated to each crop, use of irrigation and crop rotations), fertilisation and pesticide management. The second part focused on biodiversitybased techniques; for each technique, the farmer was asked (i) how he/she was implementing the techniques, (ii) if he/she was satisfied, (iii) the reason of its application, (iv) the source information or network used and (iv) to evaluate the economic impact of the technique.

\subsection{Statistical analysis}

To identify the combination of biodiversity-based techniques practised by the farmers and to further subsample the farmers, we carried out a multiple correspondence analysis (MCA), followed by a hierarchical cluster analysis on principal components (HCPC) on the sample of 194 farms. The objective of the MCA analysis was first to condense the variability between the individuals (farmers) in a few synthetic components in order to facilitate the identification of groups of individuals. The explanatory tested variables were the applied biodiversitybased techniques. To help interpret the components, we also tested supplementary qualitative (organic certification, farm location and conservation agriculture) and quantitative (rotation length, the number of cropped species in multispecies systems, the total number of cropped species, the total number of cropped species families, the total number of rotations and the total number of cover crops) variables that were not used to build the principal components. The MCA followed by the HCPC analysis performed on the first two components resulted in groups of farmers who differed in how the famers combined biodiversity-based techniques. To identify the variables that characterise each group, we obtained for each group of farmers the average level (quantitative variables) or the proportion of farmers (qualitative variables), which we tested for differences against the whole sample. If there was a significant difference, then the variable discriminated the group of individuals ( $V$ test with $p$ value $\leq 0.05$ ) and was considered to characterise the group. We tested all the biodiversity-based techniques and tested whether the supplementary variables differed between groups. These included both qualitative and quantitative data. These data were statistically tested to find the most important supplementary variables for each group ( $p$ value $\leq 0.05$ ). We considered supplementary variables to be discriminant to describe a group when $\mid V$ test $\mid \geq 2$. All statistical analyses were conducted using R (v2.15.1) (R Core Team2012) with the packages FactoMineR and ade4.

\subsection{Sub-sampling for in-depth interviews}

To better understand and characterise the diversity of practices in the farms, we selected 29 farmers from the sample with whom to carry out in-depth interviews. The farmers were located in three of the five regions previously surveyed (namely, the Rhône-Alpes, Aquitaine and Midi-Pyrénées regions). The farmers were selected within the six groups previously identified using the MCA, reaching a total of 29 farmers. We chose the farmers in order to be representative of the groups with respect to their age, academic background, principal type of farming and organic certification.

Semi-directive interviews were conducted during the spring of 2014. First, the interview was prepared on the basis of the data previously collected at the end of 2012. During the interview, information was collected related to the history of the farm and the objectives of the farmers. Then, the farmer described the logic of his/her cropping systems (choice of crops and rotation) in relation to the soil and climatic conditions, the available labour and machinery and his/her socioeconomic environment. In the final part of the interview, the farmer described his/her biodiversity-based technique applications and was asked about the benefits and constraints of each implemented technique.

For each biodiversity-based technique, the modalities of implementation by farmers were analysed. Two or three practices thus corresponded to each technique. Each practice was characterised with respect to the farm characteristics and the expectations of the farmers implementing it. For each practice, the levels of change in the farm were identified ((i) field scale, (ii) cropping system scale (rotation) or (iii) farm scale) (Navarrete et al. 2006) and classified with respect to the ESR framework of Hill and MacRae (1995). Finally, for each practice, we discussed the level of room to manoeuvre with respect to the farm characteristics.

\section{Results and discussion}

\subsection{Description of the first sample of 194 farmers}

The 194 farms surveyed had an average cropping area two to seven times larger than that of the French farm population for both conventional and organic farming systems (Table 1). 
Another specificity of the sample was the high number of farmers using reduced tillage or conservation tillage techniques ( 83 vs. $47 \%$ in the French farm population, Table 1). Most farmers of the sample (87\%) used cover crops to manage their fallow periods between the main crops compared with $30 \%$ in the French farm population (Table 1); in most cases, one $(45 \%)$ or a mix of two to four species $(42 \%)$ were sown. Although a large diversity ( $>30$ ) of cover crop species was identified in the sample, four of them were preferentially used and found in 10 to $15 \%$ of the cover crops sown: white mustard (Sinapis alba L.; 15\%), oat (Avena sativa L. and Avena strigosa Schreb.; 14\%), vetch (Vicia sativa L. and Vicia villosa L.; 12\%) and clover (Trifolium incarnatum L. and Trifolium pratense L.; $10 \%)$. One of the main motivations expressed by the farmers for using cover crops was the location of their farms on nitrate-vulnerable areas ( $61 \%$ of the sample). To meet the European regulation requirements related to nitrate pollution, they have to sow cover crops (Table 1). This technique was also widely applied because it does not require important changes at the farm scale (Dunn et al. 2016), and farmers are aware of the benefits of this technique (Arbuckle and Roesch-McNally 2015; Dunn et al. 2016). Moreover, this technique is usually associated with diverse cropping systems (Arbuckle and Roesch-McNally 2015) and large farms (Dunn et al. 2016), such as those in our sample.

The second biodiversity-based technique was the most observed technique in the sample. This technique involved the use of agroecological infrastructures ( $81 \%$ of the farms, Table 1) such as hedges (planting and/or maintenance, $73 \%$ ), buffer strips $(51 \%)$, isolated trees (planting and/or maintenance, $38 \%$ ) or even the maintenance of fallows for the wildlife (3\%). This is in line with recent European Common Agricultural Policy (CAP) incentives that favour the planting and maintenance of hedges, trees, strips and ecological focus areas (Regulations (EU) No. 1307/2013, No. 639/2014 and No. 641/2014).

The diversification of crop rotations was also largely applied in our sample, as more than $66 \%$ of the farms had a diversified crop rotation (considered in this study as a rotation with at least three different species cultivated). Consequently, the mean length of the crop rotations observed in the sample was $4.9 \pm 2.2$ years (mean $\pm \mathrm{SD}$ ) when considering only the cash crops that did not significantly differ between the five regions studied. Moreover, within those farms, only $14 \%$ had a crop rotation length $\leq 2$ years (with $4 \%$ of monoculture), $46 \%$ had a length ranging from 4 to 6 years and $21 \%$ had a rotation duration longer than 6 years. Bread wheat (Triticum aestivum L.) was the most cultivated crop and appeared in $74 \%$ of the sample crop rotations. Maize (Zea mays L.) (for grain or silage), oilseed rape (Brassica napus), barley (Hordeum vulgare L.) and sunflower (Helianthus annuus L.) were found in 50,27, 25 and $21 \%$ of the crop rotations, respectively. The most abundant Fabaceae species cultivated in the sample was alfalfa (Medicago sativa L.) followed by pea (Pisum sativum L.), which were found in 17 and $14 \%$ of the rotations, respectively. For $38 \%$ of the farms, intercropping was used and mainly consisted of cereals/legumes. Greening of the CAP also included incentives for crop diversification. The rotations of the samples were more diversified compared with those of the practices of farmers at national scale, confirming that this survey concerned innovative farms applying biodiversity-based techniques.

Agroforestry, considered here as the simultaneous cultivation of crops and trees within a field, was found in only $6 \%$ of the farms. At the national scale, the French Agroforestry Association (AFAF) recorded only 75 farms ( 425 ha) benefiting from European subsidies (2010-2013). It is likely that we overrepresented this technique in the sample compared with national data.

\subsection{MCA results: groups of farmers applying the same combination of techniques}

A two-component model was found to provide a good fit, with almost $40 \%$ of the variance explained (Fig. 2). All the correlated variables $(p<0.01)$, i.e. the biodiversity-based techniques that explain components 1 and 2 were identified and sorted according to their correlation with the components: component 1 was mainly negatively correlated with varietal mixtures, diversified rotations and cover crops, while component 2 was positively correlated with agroforestry and intercropping but negatively with agroecological infrastructures. The maximisation of the inertia gain when clustering resulted in six groups (Fig. 3).

Group 1 was composed of 12 farmers who are all applying agroforestry techniques to their farm $(p<0.001)$. Most of the farm of this "agroforestry group" (58\%) were certified organic $(p<0.01$, Fig. 3). Most of farmers of group 2 were implementing or maintaining agroecological infrastructures $(p<0.001)$ as well as implementing diversified crop rotations, varietal mixtures and cover crops. Nevertheless, these farmers did not apply intercropping $(p<0.001)$. The farms of this "diversified group" housed a higher number of crop species $(p<0.05)$ and families $(p<0.05)$ compared with the overall sample and were mainly located in northwestern France (Pays de Loire region) $(p<0.001)$ (Fig. 3). Farmers of group 3 were significantly applying intercropping $(p<0.001)$ and were mostly located in the northern part of France (Nord-Pas-de-Calais region) $(p<0.001)$. This "intercropping group" also housed a higher number of crop species $(p<0.01)$ compared with the overall sample (Fig. 3). Farmers of group 4 did not implement agroecological infrastructures $(p<0.001)$ or intercropping $(p<0.05)$; these farmers mainly implemented cover cropping $(p<0.05)$. This "no agroecological infrastructures group" was mainly located in southwestern France (Aquitaine region) $(p<0.01)$ (Fig. 3). Group 5 was denominated as the "no varietal 


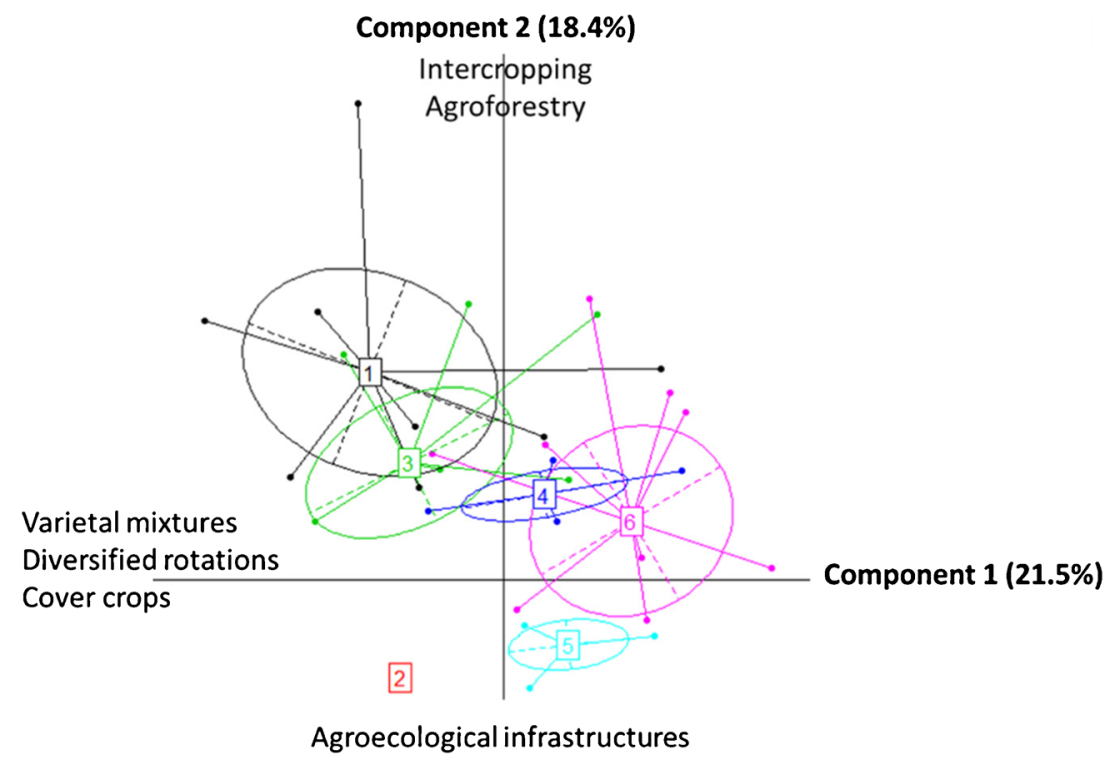

Fig. 2 Distribution and grouping of the 194 farmers who applied biodiversity-based techniques. Legend: Components 1 and 2 of the MCA explained $40 \%$ of the variance (component $1,21.5 \%$; component $2,18.4 \%)$. Component 1 is mainly negatively correlated with three techniques: application of varietal mixtures, diversified rotations and cover crops. Component 2 is mainly correlated with three techniques: negatively with agroecological infrastructures but positively with agroforestry and intercropping. The hierarchical clustering resulted in six groups of farmers. Ellipses are centred on the centroids of the groups; the width and height of the ellipses are given by the variances, and the slope of the main axis is the covariance

and/or conservation farmers are spread among the different groups. At the global sample scale, organic farmers significantly combined a greater number of techniques (mean $=3.34)$ compared with conventional farmers (mean $=2.99)(t$ test, $p<0.05)$. This finding confirms our assumption that organic farmers make a wider use of biodiversity-based techniques. When converting and further managing their farms, organic farmers usually apply a holistic approach, leading them to redesign their systems and combine a wide range of different techniques. Based on the numerous expected services of biodiversity-based techniques, organic farmers are likely to apply such techniques to cope with the lack of synthetic fertilisers and pesticides and to profit from biological and ecological processes supported by a larger biodiversity (Kremen and Miles 2012; Wezel et al. 2014). On the other hand, farmers applying conservation agricultural techniques were not significantly applying a larger number of biodiversity-based techniques. This could be explained by the fact that the main biodiversity-based techniques concerning conservation agriculture are the use of cover cropping and crop rotation diversification (complementing the reduction in soil tillage) (Peigné et al. 2007). These techniques were also two of the most implemented techniques in our sample.

Finally, the location of the farms appeared to be correlated with the different groups, highlighting that the implementation of the different biodiversity-based techniques could also be the consequence of both soil and climatic conditions and also farming system models present in the different regions of our sample. For example, group 2, where the Pays de Loire region farms were largely represented, was characterised by a larger d to organic farming, while no group was significantly re ed to conservation agriculture. This finding shows that organic 
Fig. 3 Characteristics of the 6 groups provided by the MCA followed by HCPC. Variables: explanatory, normal font; supplementary, italic font; plus sign, positively correlated; minus sign, negatively correlated; $* * * p<0.001 ; * * p<0.01$; $* p<0.05$. Green groups are more diversified groups, and orange groups are less diversified ones
Applying agroforestry

technique on the farm?

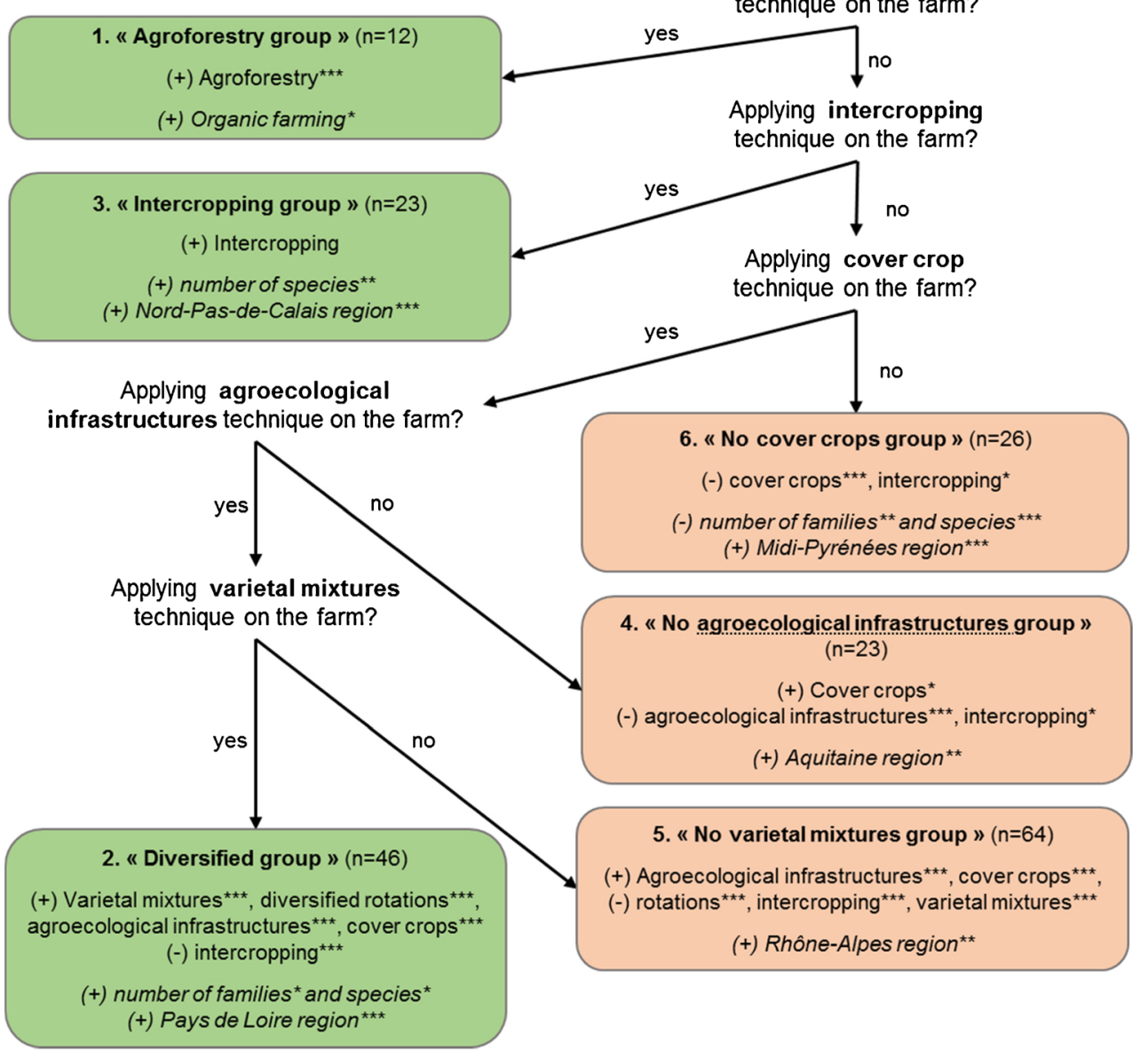

presence of agroecological infrastructures and more diversified crop rotations than in the overall sample. This is partly related to the mixed cropping/breeding farms present in the "bocage" landscape, which is quite characteristic of the western part of France. In contrast, the sampled farms of the Aquitaine region, largely present in group 4, were present in larger areas. This finding suggests that these farms partly correspond to specific farming systems, with large farms being highly specialised in some crop production (e.g. maize, carrot). This finding could explain that this group is composed of farms with low crop diversity.

\subsection{Diversity of farmer practices and motivations for their implementation}

Based on the groups resulting from the clustering (see previous section), 29 southern farmers (from the Aquitaine, MidiPyrénées and Rhône-Alpes regions) of the 194 sampled were further questioned using in-depth interviews. The number of selected farmers in each group was not exactly proportional to the total number of farms of each group. Indeed, we overrepresented farmers of the diversified groups in order to better explore the diversity of the implementation of biodiversity- based techniques (Table 2) and to further identify factors for their implementation. We interviewed three farmers from group 1, six from group 2, five from group 3, four from group 4, six from group 5 and five from group 6 . Among the 29 interviewed farmers, $31 \%$ were certified organic, $48 \%$ implemented conservation techniques, $52 \%$ managed arable farms and $48 \%$ managed mixed farms (including livestock). The size of the farms ranged from 23 to 1010 ha. Ninety-three per cent of those farmers used cover crops, $90 \%$ diversified rotations, $41 \%$ used varietal mixtures, $98 \%$ used intercropping and $10 \%$ performed agroforestry. The number of applied techniques ranged from one (a farmer in group 6) to four (farmers in group 2) per farm. Indeed, the number of applied techniques as well as the way those techniques are applied on farms depend on the expected ecosystem services and require adaptive management by farmers (Duru et al. 2015).

\subsubsection{Cover crops}

Twenty-five of twenty-nine farmers included cover crops in their crop rotations (Table 2). Most of these farmers sowed living cover crops during the long period between the harvest of a crop (generally autumn crops) and the following spring 


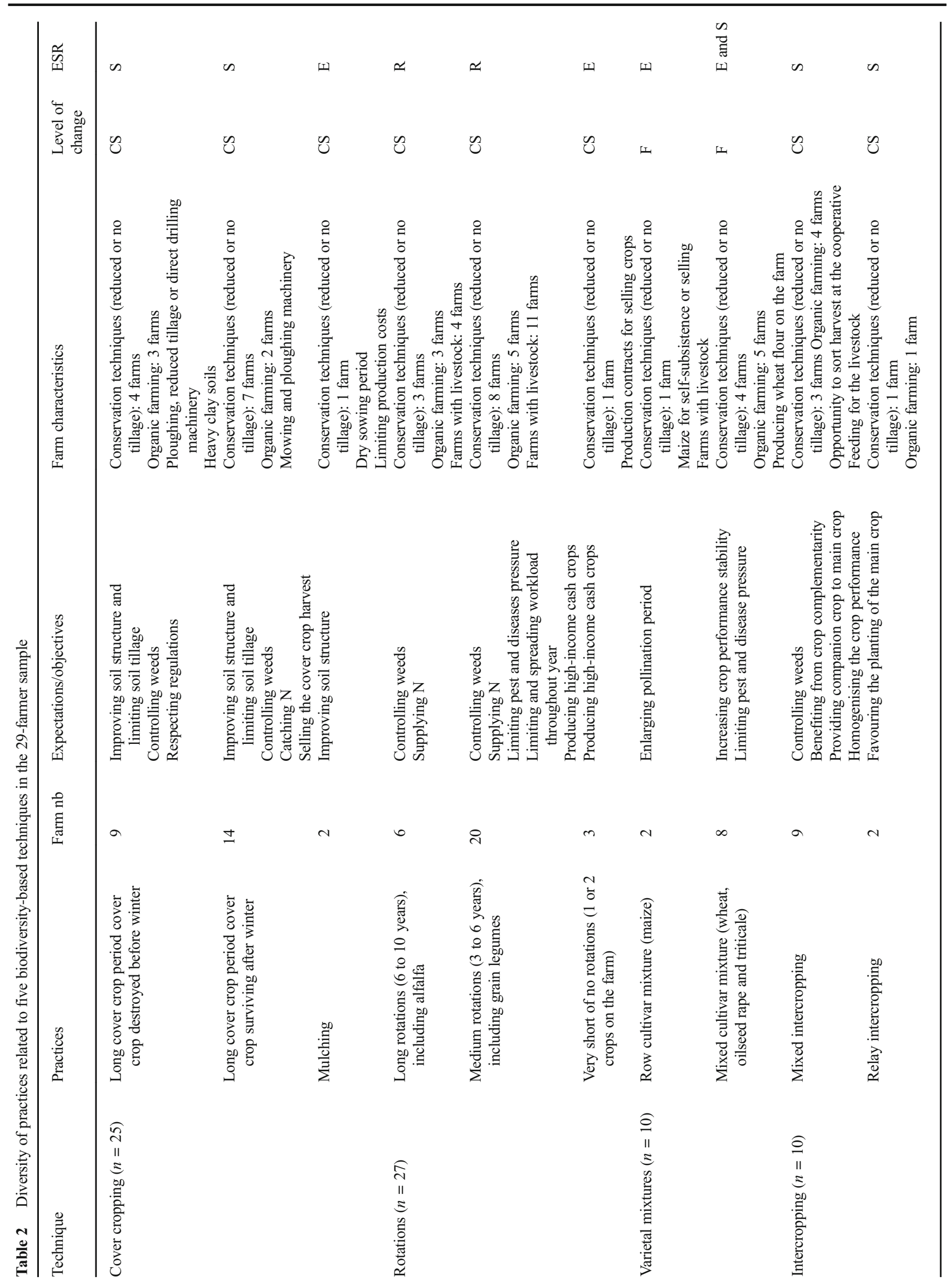




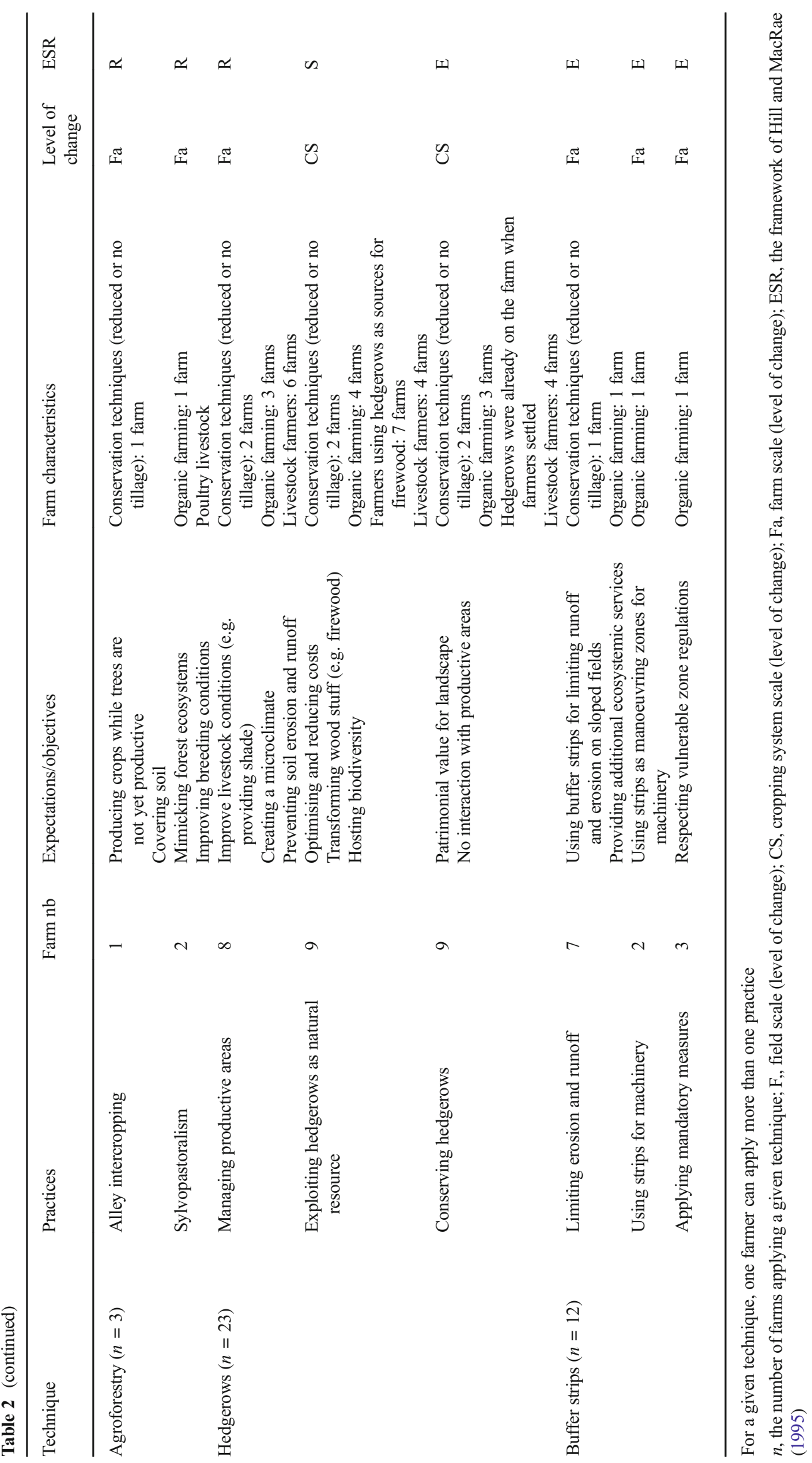


crop. Two types of living cover crop practices were identified during this long period between two cash crops: short cover crops that do not survive over winter (frost-riven species) $(n=9)$ and long cover crops that are destroyed at the end of the period between the two cash crops $(n=14)$ (Table 2). In both cases, farmers are motivated by improving soil structure and controlling weeds (thus limiting pesticide use), and this is in line with scientific studies (Schipanski et al. 2014; BlancoCanqui et al. 2015). Such practices are changing the management practices of farmers at the field and cropping system scales and imply a substitution of inputs (N, pesticides). When applying conservation techniques, cover crops cannot be destroyed with tillage operations. In the case of organic farming, the use of herbicides is banned. In both cases, farmers suggested that the ability to manoeuvre for the implementation of the technique is thus limited. In other cases, the implementation of cover crops does not require important changes in the farm; the room to manoeuvre is medium. Two farmers practised mulching of their crop residues and leaving them as ground cover during winter. In this context, this practice does not require any sowing preparation operations, and the following crop is directly sown into the mulch. The room to manoeuvre was thus qualified as high, especially when farmers use herbicides. Because there are low levels of risk associated with cover crop use (Arbuckle and RoeschMcNally 2015), farmers are likely to easily adopt this technique. Nevertheless, seed cost might limit the adoption (Dunn et al. 2016). The diversity of implementation options for cover crops affords an opportunity to match each farm specificity.

\subsubsection{Diversified crop rotations}

Three levels of diversification by increasing the length of rotations were identified: (i) long rotations (6 to 10 years), including semi-perennial crops such as alfalfa $(n=6)$; (ii) medium rotations ( 3 to 6 years) $(n=20)$, including principally annual crops; and (iii) short or no rotations (one or two crops in the farm) $(n=3)$ (Table 2). In the cases of long and medium rotations, the objectives of the interviewed farmers were first to control weeds and then supply $\mathrm{N}$ to the whole cropping system. In the case of medium rotations, farmers were also motivated by pest and disease control while simultaneously decreasing input use and limiting and spreading workloads. When applying short crop rotations or even monocultures, the motivations of farmers mostly involved the production of high-income cash crops (e.g. maize). Two farmers in our sample even combined monoculture and signed production contracts for selling their harvest. Diversified rotations (long and medium) were widely applied by organic farmers, farmers with conservation techniques and farmers with livestock. These applications occur because crop rotation is one of the pillars for sustainable functioning of both agroecosystems and conservation agriculture, especially for controlling weeds, pest and disease and for supplying $\mathrm{N}$ to the soil (Wezel et al. 2014). The diversification of rotations is also key for diversifying animal feeding and achieving self-sufficiency. Medium rotations usually include grain legumes and alternating winter and spring crops. Because increasing the number of crops in the rotation implies a strong rearrangement of cropping system management, we qualified medium and long rotation practices as redesign practices (Wezel et al. 2014) at the cropping system scale. Depending on the farm organisation and the objectives of the farmer, rotation implementation implies room to manoeuvre that ranges from medium to high, as crop rotation design is a very integrative decision-making progress that impacts farm management at multiple levels (Dury et al. 2011).

\subsubsection{Varietal mixtures}

Varietal mixtures rely on the use of intraspecific genetic diversity at the field scale for improving agroecosystem stability and function (de Vallavieille-Pope 2004; Finckh and Wolfe 2006; Lin 2011). This practice involves mixtures of cultivars that vary in many characters, including pest and disease resistance, but are sufficiently similar to be grown together. Two types of varietal mixture practices were scored in the 29farmer sample: (i) row varietal mixtures $(n=2)$, i.e. alternating rows of different varieties in the same field (observed for two farmers with maize varieties) and (ii) mixed varieties $(n=8)$, i.e. mixing varieties without specific spatial allocation for each variety (recorded for eight farmers with wheat varieties). Row varietal mixtures are used here for allogamous crops in order to lengthen the crop pollination period, allowing for increased yield and yield stability and/or for hybrid seed production. However, farmers also highlighted the modification of crop management, its components and its complexity. In the case of mixed varieties, the objective of farmers was first to better control pest and diseases and to reduce the dependency on chemical inputs. Varietal mixtures do not completely suppress or eliminate diseases. Rather, the mixtures reduce the speed of disease progress by eliminating large numbers of spores at each cycle of pathogen multiplication (de Vallavieille-Pope 2004). Given that five of these eight farmers are organic, this practice helps those farmers cope with the lack of chemical inputs, including pesticides.

Some of the interviewed farmers were also motivated to use this practice to attempt to improve flour quality. This fact was particularly true for the two farmers who processed their wheat harvest into flour and baked bread that was directly sold to consumers. Those two farmers mixed approximately 100 different wheat varieties in their fields to better adapt to soil and climate conditions and to produce high-quality flour. The benefits of varietal mixtures have been demonstrated in previous studies. For instance, field trials conducted in the UK and Denmark showed yield stability and good yield reliability 
across variable and unpredictable cropping environments (Döring et al. 2015), concluding that mixtures are less sensitive to environmental stress than are their component varieties.

As long as the farmer has the opportunity to self-process (animal feed or baking) the harvested crop or sell it, the room to manoeuvre of the varietal mixture practice is high because changes occur mainly at the field scale and do not cause important changes at the cropping system or farm scale.

\subsubsection{Intercropping}

Based on the typology proposed by Andrews and Kassam (1976), we identified two types of practices for intercropping: (i) simultaneous intercropping $(n=9)$ and (ii) relay intercropping $(n=2)$. Simultaneous intercropping refers to the joint sowing of two (or more) species in a field, while relay intercropping refers to the delayed sowing of one crop into another previously sown crop. One of these two crops could be a cover crop. The main motivation of farmers for applying simultaneous intercropping was to improve crop performance by controlling weeds, which benefits from species complementarity for natural resources use, provides a companion crop to the main crop (e.g. helping the implantation of the main crop) and stabilises crop performance among years (Table 2). Indeed, cultivating more than one species with a low-input management usually leads to higher yields compared with crops by themselves (Bedoussac et al. 2015).

Four of the nine farmers are certified organic, and three of them are applying conservation agricultural techniques. Most of these farmers are raising animals and using their intercropping harvest as feedstuff. Only two farmers are selling their harvest to a local cooperative. Indeed, sorting is often mentioned as a limit to implement the technique, as cooperatives are rarely collecting such mixtures, and individual sorting machinery is very expansive (Bedoussac et al. 2015; Duru et al. 2015; Magrini et al. 2016). Relay intercropping aims at favouring the planting of main crops while protecting the soil. Relay intercropping is being applied by one organic farmer and one conservation farmer. Because covering the soil is one of the pillars of conservation agriculture, such a practice is therefore in line with conservation principles. This practice also impacts crop management and cropping systems and helps control weeds, thus reducing input dependency ("substitution practice").

In both cases, intercropping is applied as a substitution technique to lone crops and helps improve main crop performance by reducing growth-limiting factors of lone cropping, which contributes to reduced input dependency. This technique impacts the design of rotations and, in turn, the cropping system scale. Similar to varietal mixtures, in the studied sample, the room to manoeuvre is high as long as there are opportunities for marketing, self-consumption or processing of the crop (Magrini et al. 2016). However, the opportunities for marketing species mixtures are rare and are cited as a limit by the interviewed farmers.

\subsubsection{Agroforestry}

Three of the 29 farmers sampled were applying agroforestry techniques. One of these farmers designed alley intercropping of trees with arable crops, while the other two developed sylvopastoralism systems. In the case of alley intercropping, the farmer explained that his objective was to obtain crop harvest from the alleys and to maximise soil cover while waiting for the trees to be productive. Indeed, this farmer is applying conservation agricultural techniques. The sylvopastoralism systems were developed by farmers raising poultry to improve livestock conditions. One of the farmers, who was certified organic, also expected agroforestry to mimic forest ecosystems and, in turn, provide ecosystem services, as suggested in different scientific studies (Jose 2009).

In both cases, implementing agroforestry on farms implies a long-term redesign of the cropping systems and changes the whole organisation of the farm by combining productions that were previously separate on the farm (e.g. walnut trees and arable crops or trees and livestock). Indeed, severe changes are needed, and the beneficial ecosystemic effects of agroforestry systems rely on slow processes (Rigueiro-Rodrígues et al. 2009). The associated room to manoeuvre is medium in the case of sylvopastoralism, as agroforestry requires the redesign of cropping systems and farming systems (reallocation of the production of the different plots of the farm) but does not impact farm resources. In the case of alley intercropping, the farmer is redesigning his system in steps by introducing trees to arable fields, without changing arable rotations or the irrigation system. The room to manoeuvre in this case is thus considered greater.

\subsubsection{Agroecological infrastructures}

Because agroecological infrastructures are mainly maintained on farms (hedgerows) or result from lawn applications (grass strips), we chose to briefly describe hedgerows here. Hedgerows are usually conserved when they already exist on farms. When hedgerows are planted or managed, the objectives are (i) to provide direct production services (e.g. hosting diversity or exploit firewood) or (ii) to help manage crops and animals (Table 2).

The top three biodiversity-based techniques identified in our 29 interviews were crop rotations $(93 \%)$, cover crops $(86 \%)$ and agroecological infrastructure (79\%), which is consistent with the three main techniques of the 194 samples. Each practice was classified according to ESR framework of Hill and MacRae (1995) (Table 2): $31 \%$ involved improving input use (efficiency), 37\% were relevant to alternative techniques (substitution) and $32 \%$ required a redesign of the whole 
cropping or farming system. Most of these biodiversity-based techniques were applied at the cropping system scale (71.6\%), and the room to manoeuvre was mainly considered high. This could be explained by the high percentage of farms that are involved in organic farming or conservation agriculture and by the presence of livestock in 14 farms.

\subsection{Constraints and factors of the applications on farms}

\subsubsection{Internal and external factors favouring the application of biodiversity-based techniques}

The second series of in-depth interviews with farmers highlighted various lock-in processes that could slow down the application of such biodiversity-based techniques in cropping systems. Four main factors favouring the application of biodiversity-based techniques on farms have been identified. Each factor applies to each of the studied biodiversitybased techniques, but the effect of the factors on technique application varies depending on the technique considered.

The two first factors are intrinsic to the farming system and depend on (i) the labour force and (ii) machinery availability on the farm. Available worktime and crop management planning were often noted as limitations to the application of biodiversity-based techniques. Six of the twenty-nine interviewed farmers identified periods of heavy workload during the year, and these workloads prevented the farmers from introducing some of these techniques. Indeed, several farmers suggested that these techniques may induce a higher monitoring requirement (e.g. introduction of a new crop in the crop rotation); rather, the farmers preferred to master their overall farm management than introduce new techniques. Farmers often fear that the introduction of new crops will increase total labour requirements (Liebman and Schulte 2015), increase periods of heavy workload or delay harvest periods from the optimum (Meynard et al. 2013). It is then interesting to note that in our sample of 29 farms, farmers with hired labour forces implemented more of these biodiversity-based techniques than did the other farmers. Similarly, another farmer mentioned that the diversification of his crop rotation led to a reduction of his free time, although this reduction was associated with a better spreading of workload throughout the year. When dealing with intercropping techniques, some farmers highlighted the additional time needed for grain sorting. The introduction of cover crops, particularly during the summer period (after a winter crop), appears to be the least constraining technique in terms of workload, according to the interviewed farmers. Indeed, cover crop implementation increases work requirements during the sowing period but does not require any operations during the cover crop cycle until its destruction.

The second intrinsic factor is the need for specific machinery. Most of the interviewed farmers specifically mentioned the need to invest in seeding and sorting equipment for the application of some biodiversity-based techniques. Six of the interviewed farmers said that they could not implement some of these techniques because of a lack of equipment and the difficulty to have a return on investment using that equipment. As a consequence, more than half of the interviewed farmers (16/29) joined a farm machinery cooperative to share the costs and mainly use the seeding equipment. Other farmers $(9 / 29)$ preferred to invest individually and own machinery to avoid competing for the use of machinery with other farmers during the periods of intense work. Finally, sorting equipment seems necessary to develop intercropping techniques for grain production. Indeed, the main constraint against this technique appears to be that industries and cooperatives do not accept harvested grain mixtures (Bedoussac et al. 2015; Magrini et al. 2016), although this was not the case for one of the interviewed farmers. Consequently, except the use of intercrop production for feeding their own livestock, farmers who developed this technique invested in individual sorting equipment (6/29) and sold both productions separately. Five farmers were interested in implementing such techniques but could not because of the lack of sorting equipment.

The other two factors concerned external factors linked to the socio-economic environment of the farm: (iii) market opportunities and (iv) networking. First, the diversification of cultivated crops implies the development of new market outlets. Most of the interviewed farmers (25/29) sell their crops to cooperatives or traders who do not accept varietal or crop mixtures. Livestock farmers have the opportunity to directly use their production for animal feeding, but the type of animal production may largely influence the possible crops and mixtures. Livestock farmers introduced mixed crops (four farmers) and temporary grassland (alfalfa) into the crop rotations. Nevertheless, in our sample of 29 farmers, no significant difference was found in the number of crops between cereal or mixed farms. Five farmers directly sell their production, creating their market outlet. In those cases, production is generally processed on farm (e.g. flour, bread, pastas, oil, popcorn) to increase profit. This factor seems to be a powerful way to unlock both the introduction of new crops that are usually not collected by cooperatives into the rotation (e.g. lentil, flaxseed or spelt) and the application of biodiversity-based techniques (mainly intercropping and cultivar mixtures). However, these farmers face an increased workload required for developing, selling and processing activities.

Sharing information between farmers through networks favours the diffusion and application of biodiversity-based techniques. The diversification of crop production usually implies the redesign of cropping systems and rotations, involving complex crop management and requiring intensive skills and knowledge to crop a larger number of species (Navarrete et al. 2014). This increase in the cognitive load and the associated need for information and learning new methods were also 
identified in the change towards more sustainable practices (Chantre and Cardona 2014). Consequently, the presence of a structured network may largely facilitate the spread of these techniques, due to references and advice provided (Chantre and Cardona 2014), and sometimes this spread is also driven by the generated confidence of not being alone. Confrontation regarding the experiences of farmers is a way to optimise the practice to the specific conditions of the farm and strengthen the idea that the farmer is using proper techniques. Ten of the twenty-nine interviewed farmers belonged to the BASE network, which aims at developing conservation agriculture in France. According to the farmers, the BASE network provided useful information that helped them change their perception of soil-plant interactions and favour the application of techniques that protect and stimulate soil activity. These paradigm changes imply an increase in the length and diversity of their crop rotations and the large adoption of cover cropping techniques. Similarly, five other farmers belonged to Dephy Ecophyto network farms, which are supported by the French government, to find technical options to reduce pesticide use. These farmers highlighted how this network provided them information and advice (mainly provided by chambers of agriculture in France) to change their practices. In our case, particular cover cropping and diversified crop rotations were favoured. Finally, one farmer belonged to a network promoting agroforestry and hedge establishment.

\subsubsection{Effects of farming systems on the application of biodiversity-based techniques}

The second series of in-depth interviews with farmers confirmed the specificity of the application of biodiversity-based techniques with respect to farming systems. Indeed, three characteristics of farming systems might explain the way farmers implemented these techniques: (i) organic farming certification, (ii) conservation agriculture application and (iii) livestock breeding.

First, the organic farmers in our sample applied more diversity-based techniques (mean $=3.34$ ) than did the conventional farmers in our sample (mean $=2.99$ ), which confirmed one of our assumptions. Organic farmers were also more likely to apply some of the biodiversity-based techniques than were other farmers. This finding could be the consequence of a different philosophy of life, causing organic farmers to establish an environmentally friendly form of agriculture often associated with agro-biodiversity (Malézieux et al. 2009). This finding also suggests that the lack of chemical inputs, which organic farmers are faced with, makes difficult the use of remedial techniques, as organic inputs might be more expensive and less efficient (Bàrberi 2002; David et al. 2005). This phenomenon may encourage organic farmers to diversify practices and increase agro-biodiversity, and these farmers could profit from biological and ecological processes providing agro-ecological services to the system, including both better control of weeds, pests and diseases (Ratnadass et al. 2012) and an increased soil fertility (Wezel et al. 2014). For example, organic farmers were generally applying medium-to-long crop rotations (i.e. more than 6 years) and presented greater numbers of species in their cropping systems. Such crop rotations, which included different species and alternating winter and spring crops, have shown their efficiency in reducing weed infestation as well as pest and disease contamination (Colbach et al. 1997; Bàrberi 2002). Some techniques (e.g. crop and/or varietal mixtures) were less applied in our samples but were applied relatively more by organic farmers. Some organic farmers of our sample developed innovative market outlets and more direct selling and onfarm transformation, notably to compensate for a general decrease in crop productivity by higher added value and sales margins. This also favours the diversification of crops on the farm to increase the number of products sold on farm. In contrast with other techniques, organic farming makes difficult the application of cover cropping, particularly during long intercrop periods, as the interviewed farmers often mentioned the difficulty to destroy the cover without herbicides (Casagrande et al. 2016). This prompts organic farmers to avoid such techniques or adapt cover crops of different species that are sensitive to frost or more easily mechanically destroyed.

Cover cropping techniques are also favoured by conservation agriculture, but the techniques of farmers belonging to this movement (BASE network) did not significantly differ from those of other farmers in our larger sample; these farmers implementing conservation agriculture, did not apply significantly more biodiversity-based techniques compared with the others. This finding might be the consequence of our sampling method, which aimed at identifying innovative farmers who apply biodiversity-based techniques. As such, the farmer sample was not always representative of French farmers. In our samples, the most applied techniques were cover cropping and the diversification of the crop rotations. Both are pillars of conservation agriculture. Therefore, these findings could explain that these conservation farmers did not differentiate from other farmers in our sample.

Finally, the breeders in our sample (16/29 and 83/194 for the largest sample) were applying all the studied biodiversitybased techniques. The breeders did not significantly differ in the numbers or types of applied techniques compared with the overall sample. Nevertheless, interviews highlighted that livestock presence on the farm can facilitate the implementation of some of the techniques. Thus, as a well-known example, breeders favoured crop and varietal mixtures for animal feeding. Indeed, some of the breeders mentioned that sorting grains was not necessary. Twelve of the sixteen interviewed breeders specifically mentioned that livestock feeding offers the opportunity to promote some crops in the crop rotation 
(e.g. fodder and cover crops or crop mixtures), while crops that were easier to market were sold. Livestock farmers also considered that the diversification of crop rotations and the production of manure were ways to increase the productivity of these cash crops. Nevertheless, the results depend on the type of animal production. Indeed, the breeders of monogastric animals (i.e. poultry and rabbit here) specified that they were not able to valorise such mixtures, as well as on-farm production in general, for animal feeding, as these breeders underlined how difficult it was to evaluate the composition of the mixtures for daily rations.

In conclusion, the farming system does not seem to have a major impact on the type of biodiversity-based techniques applied by farmers. Nevertheless, the farming system impacts the transference of these techniques into practices, providing a diversity of applications for a given technique.

\section{Conclusion and perspectives}

This paper aimed at describing biodiversity-based techniques and their implementation on farms. We tracked "innovative" farms that applied such techniques to gain insight into the practices of these farms and the factors limiting or favouring the development of biodiversity-based techniques. We showed that the most frequent techniques applied were cover crops, agroecological infrastructures and the diversification of crop rotation. The farmers had different profiles of diversification, depending on the number and combination of techniques, ranging from simple compliance with European regulations (e.g. cover crop application) to complex redesigned systems (e.g. agroforestry). Organic farms significantly combined more biodiversity-based techniques compared with the overall sample, implying a systemic redesign of the farming system. Surprisingly, the conservation agriculture farmers in our sample did not apply more of these techniques and seemed to mainly focus on cover cropping. In actuality, the availability of both labour force and specialised machinery (internal factors) as well as access to market opportunities and the exchange of knowledge through networking (external factors) could favour the development of biodiversity-based techniques on farms.

To achieve the objectives of the French government and to increase the number of agroecological farms, attention should be paid to designing adequate policies that favour biodiversitybased techniques. Such policies should help unlock the limiting factors of their implementation by favouring access to specialised machinery (e.g. sorting equipment) and by supporting the development of new market outlets for both the diversification of crops (e.g. improve the sorting capacities of cooperatives) and the development and structuration of networks. It is crucial to support not only the direct selling of food systems but also long-chain food systems to significantly increase the implementation of biodiversity-based techniques. Moreover, additional research is needed to (i) better understand the effects of the techniques and, in particular, the combinations of these techniques in various pedoclimatic contexts, thus limiting the required monitoring time and providing information useful to farmers; (ii) improve sorting machinery technology and its efficiency and operating speed; and (iii) analyse the lock-in of the whole agrifood system.

Acknowledgements The authors would also like to thank the students from EI Purpan, ISARA-Lyon, Groupe ESA (Angers), and ISA Lille, who helped carry out phone interviews and interviewed the farmers who kindly participated in this survey.

Funding information This work was funded by the Fédération des Ecoles Supérieures d'Ingénieurs en Agriculture (FESIA) and the Groupama under the programme "FESIA AgroEco-Filières".

\section{References}

Agreste (2010) RA 2010

Agreste (2014) Enquête Pratiques culturales 2011 Principaux résultats. Agreste Les Dossiers 21:23-26

Altieri M, Funes-Monzote FR, Petersen P (2011) Agroecologically efficient agricultural systems for smallholder farmers: contributions to food sovereignty. Agron Sustain Dev. https://doi.org/10.1007/ s13593-011-0065-6

Andrews DJ, Kassam AH (1976) The importance of multiple cropping in increasing world food supplies. Am Soc Agron 1-10

Arbuckle JG, Roesch-McNally G (2015) Cover crop adoption in Iowa: the role of perceived practice characteristics. J Soil Water Conserv 70:418-429. https://doi.org/10.2489/jswc.70.6.418

Bàrberi P (2002) Weed management in organic agriculture: are we addressing the right issues? Weed Res 42:177-193. https://doi.org/10. 1046/j.1365-3180.2002.00277.x

Bedoussac L, Journet E-P, Hauggaard-Nielsen H et al (2015) Ecological principles underlying the increase of productivity achieved by cereal-grain legume intercrops in organic farming. A review. Agron Sustain Dev 35:911-935. https://doi.org/10.1007/s13593014-0277-7

Blanco-Canqui H, Shaver TM, Lindquist JL et al (2015) Cover crops and ecosystem services: insights from studies in temperate soils. Agron J 107:2449-2474. https://doi.org/10.2134/agronj15.0086

Casagrande M, Peigné J, Payet V et al (2016) Organic farmers' motivations and challenges for adopting conservation agriculture in Europe. Org Agric 6:281-295. https://doi.org/10.1007/s13165015-0136-0

Chantre E, Cardona A (2014) Trajectories of French field crop farmers moving toward sustainable farming practices: change, learning, and links with the advisory services. Agroecol Sustain Food Syst 38: 573-602. https://doi.org/10.1080/21683565.2013.876483

Colbach N, Lucas P, Cavelier N, Cavelier A (1997) Influence of cropping system on sharp eyespot in winter wheat. Crop Prot 16:415-422. https://doi.org/10.1094/phyto.1997.87.1.26

David C, Jeuffroy MH, Laurent F et al (2005) The assessment of AzodynOrg model for managing nitrogen fertilization of organic winter wheat. Eur J Agron 23:225-242. https://doi.org/10.1016/j.eja. 2004.08.002

Döring TF, Annicchiarico P, Clarke S et al (2015) Comparative analysis of performance and stability among composite cross populations, 
variety mixtures and pure lines of winter wheat in organic and conventional cropping systems. F Crop Res 183:235-245. https://doi. org/10.1016/j.fcr.2015.08.009

Dunn M, Ulrich-Schad JD, Prokopy LS et al (2016) Perceptions and use of cover crops among early adopters : findings from a national survey. J Soil Water Conserv 71:29-40. https://doi.org/10.2489/ jswc.71.1.29

Duru M, Therond O, Martin G et al (2015) How to implement biodiversity-based agriculture to enhance ecosystem services: a review. Agron Sustain Dev. https://doi.org/10.1007/s13593-0150306-1

Dury J, Schaller N, Garcia F et al (2011) Models to support cropping plan and crop rotation decisions. A review. Agron Sustain Dev. https:// doi.org/10.1007/s13593-011-0037-x

Finckh MR, Wolfe MS (2006) Diversification strategies. In: Cooke BM, Gareth Jones D, Kaye B (eds) The epidemiology of plant diseases, 2nd edn. Springer, Netherlands, pp 269-307

Hill SB, MacRae RJ (1995) Conceptual framework for the transition from conventional to sustainable agriculture. J Sustain Agric 7:81-87. https://doi.org/10.1300/J064v07n01_07

Jose S (2009) Agroforestry for ecosystem services and environmental benefits: an overview. Agrofor Syst 76:1-10. https://doi.org/10. 1007/s10457-009-9229-7

Kremen C, Miles A (2012) Ecosystem services in biologically diversified versus conventional farming systems: benefits, externalities, and trade-offs. Ecol Soc 17:25. https://doi.org/10.5751/ES-05035170440

Liebman M, Schulte LA (2015) Enhancing agroecosystem performance and resilience through increased diversification of landscapes and cropping systems. Elem Sci Anthr 3:41. 10.12952/journal.elementa. 000041

Lin BB (2011) Resilience in agriculture through crop diversification: adaptive management for environmental change. Bioscience 61: 183-193. https://doi.org/10.1525/bio.2011.61.3.4

Magrini M-B, Anton M, Cholez C et al (2016) Why are grain-legumes rarely present in cropping systems despite their environmental and nutritional benefits? Analyzing lock-in in the French agrifood system. Ecol Econ 126:152-162. https://doi.org/10.1016/j.ecolecon. 2016.03.024

Malézieux E, Crozat Y, Dupraz C et al (2009) Mixing plant species in cropping systems: concepts, tools and models. A review. Agron Sustain Dev 29:43-62. https://doi.org/10.1051/agro:2007057

Meynard J-M, Messéan A, Charlier A, et al (2013) Freins et leviers à la diversification des cultures. Etude au niveau des exploitations agricoles et des filières. Synthèse du rapport d'étude

Naudin C, van der Werf HMG, Jeuffroy M-H, Corre-Hellou G (2014) Life cycle assessment applied to pea-wheat intercrops: a new method for handling the impacts of co-products. J Clean Prod 73: 80-87. https://doi.org/10.1016/j.jclepro.2013.12.029

Navarrete M, Le Bail M, Papy F et al (2006) Combining leeway on farm and supply basin scales to promote technical innovations in lettuce production. Agron Sustain Dev 26:77-87. https://doi.org/10.1051/ agro:2005062

Navarrete M, Dupré L, Lamine C (2014) Crop management, labour organization, and marketing: three key issues for improving sustainability in organic vegetable farming. Int J Agric Sustain 13:257-274. https://doi.org/10.1080/14735903.2014.959341

Peigné J, Ball BC, Roger-Estrade J, David C (2007) Is conservation tillage suitable for organic farming? A review. Soil Use Manag 23: 129-144. https://doi.org/10.1111/j.1475-2743.2006.00082.x

Ponisio LC, M'Gonigle LK, Mace KC et al (2015) Diversification practices reduce organic to conventional yield gap. Proc R Soc B Biol Sci 282:20141396-20141396. https://doi.org/10.1098/rspb.2014. 1396

Ratnadass A, Fernandes P, Avelino J, Habib R (2012) Plant species diversity for sustainable management of crop pests and diseases in agroecosystems: a review. Agron Sustain Dev 32:273-303. https:// doi.org/10.1007/s13593-011-0022-4

Rigueiro-Rodrígues A, McAdam J, Mosquera-Losada M (2009) Agroforestry in Europe. Current status and future prospects. Springer, Dordrecht

Salembier C, Elverdin JH, Meynard J-M (2016) Tracking on-farm innovations to unearth alternatives to the dominant soybean-based system in the Argentinean Pampa. Agron Sustain Dev 36:1-10. https:// doi.org/10.1007/s13593-015-0343-9

Schipanski ME, Barberchack M, Douglas MR et al (2014) A framework for evaluating ecosystem services provided by cover crops in agroecosystems. Agric Syst 125:12-22. https://doi.org/10.1016/j. egypro.2016.11.209

Tilman D, Cassman KG, Matson PA et al (2002) Agricultural sustainability and intensive production practices. Nature 418:671-677. https:// doi.org/10.1038/nature01014

Tomich TP, Brodt S, Ferris H et al (2010) Agroecology: a review from a global-change. Annu Rev Environ Resour 36:193-222. https://doi. org/10.1146/annurev-environ-012110-121302

de Vallavieille-Pope C (2004) Management of disease resistance diversity of cultivars of a species in single fields: controlling epidemics. C R Biol 327:611-620. https://doi.org/10.1016/j.crvi.2003.11.014

Wezel A, Casagrande M, Celette F et al (2014) Agroecological practices for sustainable agriculture. A review. Agron Sustain Dev 34:1-20. https://doi.org/10.1007/s13593-013-0180-7

Willey RW (1979) Intercropping its importance and research needs part 1. Competition and yield advantages 32 